\title{
Paradoxical Low Flow Low Gradient Severe Aortic Stenosis-An Under-Recognized Entity
}

\author{
Phillip Crane ${ }^{1,2}$, Mandeep Singh Kalsi',2, Matias Yudi², Naveen Sharma ${ }^{1}$ \\ ${ }^{1}$ Department of Cardiology, The Northern Hospital, Melbourne, Australia \\ ${ }^{2}$ Department of Cardiology, Austin Health, Melbourne, Australia \\ Email: phillip.crane@nh.org.au
}

How to cite this paper: Crane, P., Kalsi, M.S., Yudi, M. and Sharma, N. (2022) Paradoxical Low Flow Low Gradient Severe Aortic Stenosis-An Under-Recognized Entity. Case Reports in Clinical Medicine, 11, 31-36.

https://doi.org/10.4236/crcm.2022.112006

Received: January 19, 2022

Accepted: February 25, 2022

Published: February 28, 2022

Copyright $\odot 2022$ by author(s) and Scientific Research Publishing Inc. This work is licensed under the Creative Commons Attribution International License (CC BY 4.0).

http://creativecommons.org/licenses/by/4.0/

\begin{abstract}
Some patients with severe aortic stenosis (AS), due to restrictive cardiac physiology, paradoxically have relatively low flow and low gradients across stenotic aortic valves despite preserved left ventricular (LV) systolic function. It results in symptoms and reduced quality of life and carries a high mortality. Whilst this form of severe AS, termed paradoxical low flow low gradient (pLFLG), is well reported, patients with this diagnosis experience inappropriate barriers to aortic valve replacement (AVR), the only efficacious treatment. We present the case of an 88-year-old female with 12 months of exertional dyspnoea on a background of hypothyroidism and hypercholesterolemia. Transthoracic echocardiogram (TTE) revealed LV hypertrophy, with a small LV cavity size and reduced stroke volume, yet normal systolic function. A heavily calcified aortic valve was identified with severe aortic stenosis, based on valve area, yet with incongruous mean transvalvular gradient of $25 \mathrm{mmHg}$ (severe $\geq 50 \mathrm{mmHg}$ ). Following exclusion of other differential diagnoses, her symptoms were attributed to paradoxical LFLG severe AS. She was however declined definitive transcatheter aortic valve implantation (TAVI) due to her paradoxically low mean aortic gradient. Following further deterioration in her symptoms and supportive quantification of poor exercise performance, she was ultimately re-referred, accepted, and underwent TAVI. Following her AVR, the patient experiences significant improvement in both symptoms and quality of life after only one month. Paradoxical LFLG severe AS remains a well-documented yet under recognized disease. It carries high morbidity and mortality if untreated, yet is significantly less likely to be referred and accepted for intervention. With its prevalence expected to rise with an ageing population, this case serves as a timely reminder for clinicians to address the under recognition of important pathology.
\end{abstract}

\section{Keywords}

Paroxysmal Low Flow Low Gradient, Doppler Echocardiography, Aortic 
Valve Stenosis, Aortic Valve Implantation, Valvular Heart Disease

\section{Introduction}

Aortic Stenosis is one of the most common and serious valvular disorders of the heart. It is a progressive, pathological narrowing of the aortic valve resulting in a hemodynamically significant left ventricular outflow tract obstruction. When untreated it results in characteristic symptoms of exertional angina, syncope and heart failure. Whilst genetic, anatomical and clinical factors can contribute to the development of aortic stenosis, its progression to severe disease is associated with male sex, age, and the severity of narrowing and degree of aortic valvular calcification [1].

Severe AS is a highly symptomatic and functionally limiting disease with a poor prognosis, mortality greater than $50 \%$ within 2 years [1]. Aortic valve replacement (AVR), surgical or transcatheter, is the only effective treatment, and is a Class I indication for severe AS with symptoms and/or systolic heart failure, with reduced LV ejection fraction [2]. Severe AS is defined by an indexed aortic valve area (iAVA) $\leq 0.6 \mathrm{~cm}^{2} / \mathrm{m}^{2}$, a mean transvalvular pressure gradient (MPG) $\geq$ $40 \mathrm{mmHg}$ and dimensionless index of less than 0.25 . However, some patients have discordantly low MPG, in the setting of reduced, termed low-flow, low-gradient (LFLG), or preserved, termed paradoxical LFLG (pLFLG), LV systolic function. Outcomes for patients with pLFLG AS are at least as dire [3] [4] as patients with traditional high-gradient severe AS, yet are significantly less likely to be referred for and accepted for intervention [5] [6].

\section{History of Presentation}

We present the case of an 88-year-old female initially referred to a cardiologist with progressive exertional dyspnea, particularly upon walking up inclines.

\subsection{Past Medical History}

She was otherwise well and independent with a past history of hypothyroidism and dyslipidemia. She has minimal cardiovascular risk factors, was a lifelong non-smoker with no known cardiac or respiratory pathology.

On examination, she was normotensive, blood pressure $116 / 80 \mathrm{mmHg}$, with a regular heart rate of 78 beats per minute. She has a BMI of $22.92 \mathrm{~kg} / \mathrm{m}^{2}$ and a small BSA of $1.54 \mathrm{~m}^{2}$. She has a soft ejection systolic murmur at the right upper sternal edge radiating to her carotids, consistent with moderate AS without evidence of cardiac failure. ECG revealed sinus rhythm, left axis deviation and left bundle branch block.

\subsection{Investigations}

A chest x-ray showed moderate cardiomegaly and no evidence of cardiac failure or lung pathology. Respiratory causes for her dyspnoea were excluded with normal 
pulmonary function tests, HRCT and connective tissue screen. She underwent coronary angiography and echocardiography to investigate cardiac aetiology.

On TTE she had normal LV size and systolic function, a small LV cavity with a reduced stroke volume, indexed to BSA, at $29 \mathrm{ml} / \mathrm{m}^{2}\left(\mathrm{~N}>35 \mathrm{ml} / \mathrm{m}^{2}\right)$. Her aortic valve $(\mathrm{AV})$ was heavily calcified, with two-dimensional appearance of moderate to severe stenosis (Video: https://youtu.be/Yr-qSiYWJiY). Doppler derived AV measurements were discordant, MPG of $25 \mathrm{mmHg}$ (severe $\geq 40 \mathrm{mmHg}$ ), peak jet velocity $3.3 \mathrm{~m} / \mathrm{s}$ (severe $\geq 4 \mathrm{~m} / \mathrm{s}$ ) and dimensionless index of 0.3 (severe $<0.25$ ), in contrast to an iAVA of $0.52 \mathrm{~cm}^{2} \mathrm{~m}^{2}$ (severe $<0.6 \mathrm{~m}^{2} \mathrm{~m}^{2}$ ), indicative of severe stenosis (Figure 1). She has no other significant valvular pathology. A transesophageal echocardiogram was performed yet added little due to significant calcium artifact. Following exclusion of cardiac ischaemia via angiography (demonstrating FFR negative, moderate stenosis of the left anterior descending artery), her dyspnoea was attributed to pLFLG severe AS. Her NT-proBNP was with in normal limits.

\subsection{Management}

She was referred for and discussed in a multidisciplinary cardiac surgical meeting for consideration of transcatheter aortic valve implantation (TAVI). Intervention was declined on the grounds that her MPG indicated her AS was not severe account for cause her symptoms.

With progressive dyspnea, she underwent functional testing to quantify her exercise limitation. Exercise testing was however aborted due to profound dyspnoea on minimal exertion and was complicated by a type 2 myocardial infarction evidenced by a mild Troponin I elevation to $67 \mathrm{ng} / \mathrm{L}(\mathrm{N}<48 \mathrm{ng} / \mathrm{L})$. A follow-up 6-minute walk test (6 MWT) of $175 \mathrm{~m}$ demonstrated severely restricted age-matched exercise capacity $(\mathrm{N}>550 \mathrm{~m})$.

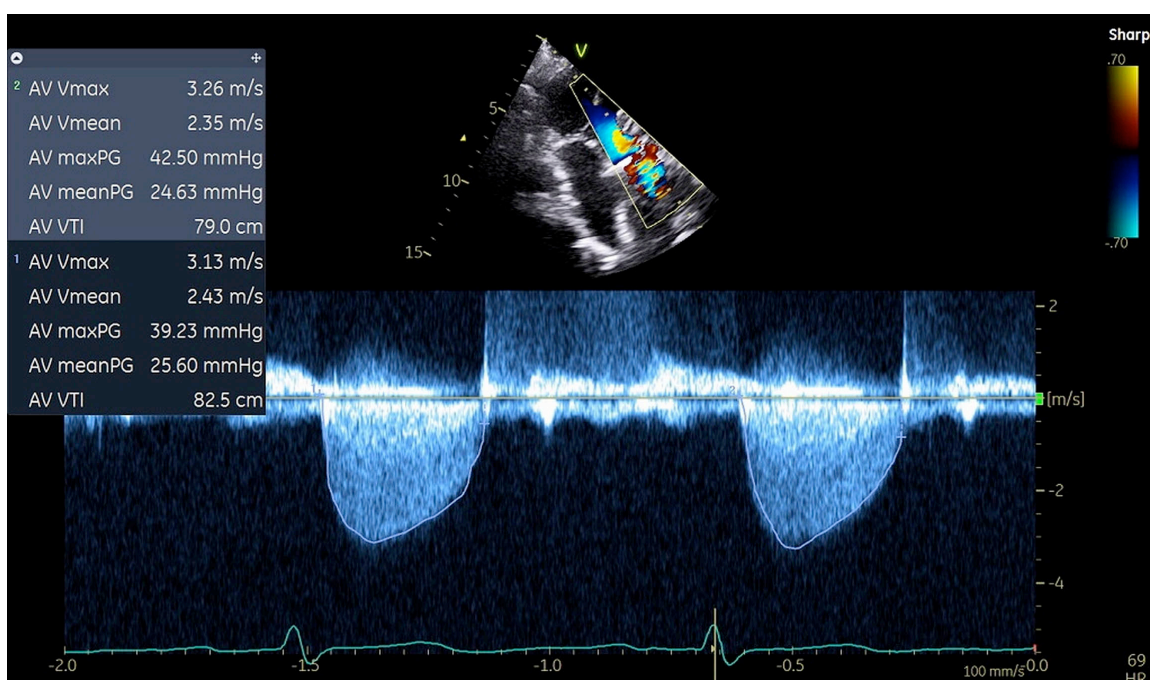

Figure 1. Continuous Wave Doppler of the Aortic Valve: Apical 3 chamber view with doppler measurements demonstrating jet velocity and MPG consistent with moderate stenosis. 
With quantitative demonstration of severe functionally AS, she was re-referred and accepted for TAVI. She received a $26 \mathrm{~mm}$ Evolut PRO (Medtronic, Minneapolis, Minnesota) AV, with an uncomplicated procedure and recovery. At 6-week review, patient reported significant symptomatic and quality of life improvement, with a corresponding $100 \mathrm{~m}$ improvement in her 6 MWT.

\section{Discussion}

Paradoxical LFLG is a severe form of AS with paradoxically low aortic transvalvular gradient in the presence of preserved LV systolic function. The low flow state in pLFLG AS results from restrictive cardiac physiology, characterized by concentric LV hypertrophy with reduced cavity size and compliance [6] [7]. Accounting for up to $35 \%$ of severe AS [7], the prevalence of pLFLG AS is expected to rise with an ageing population. Factors associated with an ageing population contribute to the pathogenesis of the restrictive cardiac physiology of pLFLG AS yet are incompletely understood. These factors include increasing age, female sex, hypertension, type 2 diabetes, atrial fibrillation, coronary heart disease, as well as an increased cumulative burden of cardiovascular comorbidities [3]. For our patient, her gender and age were the only risk factors for pLFLG AS identified.

The impact of pLFLG AS on quality of life and prognosis must not remain underappreciated. It is highly symptomatic with up to $70 \%$ mortality at 2 years if treated medically [4] [6]. Aortic valve replacement (AVR), surgical or transcatheter, is the gold standard treatment for all forms of symptomatic severe AS. Following AVR, pLFLG AS patients experience significant improvement in symptoms and quality of life, and a quantitative increase in their exercise capacity [8]. However, despite a 30\% reduction in all-cause mortality following AVR [9], mortality in pLFLG AS patients remains high, reflecting the increased risk profile of this subgroup. Age remains an independent risk factor for poor patient prognosis pre and posts AVR [3] emphasizing the need for early diagnosis and prompt referral for intervention.

Despite the poor and time critical outcomes of pLFLG AS, these patients are less likely to be referred and accepted for AVR when compared with high MPG severe AS [7] [10]. A combination of misdiagnosis, underappreciation of the impact of low-flow states, and overreliance on MPG is likely responsible. Whilst the updated 2017 ESC/EACTS valvular heart disease guidelines [2] reinforced flow status and stroke volume in the diagnostic algorithm of severe AS, it is clear that this approach has been incompletely adopted. It is vital for clinicians encountering symptomatic patients with otherwise considered non-severe AS and low MPG, to be thoroughly investigated for pLFLG AS utilizing an integrated approach including functional testing, cardiac biomarkers, CT calcium score, TTE and cardiac MRI. Furthermore, once diagnosed it is imperative to advocate on behalf of patients if, and perhaps more likely when, met with resistance to definitive intervention. 
Our case highlights important challenges when treating patients with pLFLG AS. The diagnosis is complex and requires a multi-modality approach to delineate clinical finding and correlate imaging with symptoms and functional capacity. Increased understanding of this entity amongst general clinicians and referral to a collaborative multi-disciplinary heart teams are needed to address the barriers to intervention many patients experience. This case demonstrates the inappropriate rejection of, and unnecessary delays to, AVR contributed to by fixed notions regarding AS severity. Given the patient demographic and time critical outcomes, pLFLG AS patients are subgroup of AS in which delays to surgery can be least afforded, let alone the associated ongoing reduction in quality of life, and potential increased care needs and caregiver burden, that patients and family experience. Furthermore, given the projected increased prevalence of disease states associated with pLFLG AS due to an ageing population, this case is an important reminder of this well documented but poorly recognized disease.

\section{Funding and Disclosures}

Nil sources of funding were utilized and authors report no relationship with industry or conflicts of interest.

\section{Conflicts of Interest}

The authors declare no conflicts of interest regarding the publication of this paper.

\section{References}

[1] Otto, C.M. and Prendergast, B. (2014) Aortic-Valve Stenosis-from Patients at Risk to Severe Valve Obstruction. The New England Journal of Medicine, 371, 744-756. https://doi.org/10.1056/NEJMra1313875

[2] Baumgartner, H., Falk, V., Bax, J.J., De Bonis, M., Hamm, C., Holm, P.J., et al. (2017) ESC/EACTS Guidelines for the Management of Valvular Heart Disease. European Heart Journal, 38, 2739-2791. https://doi.org/10.1093/eurheartj/ehx391

[3] Clavel, M.A., Dumesnil, J.G., Capoulade, R., Mathieu, P., Senechal, M. and Pibarot, P. (2012) Outcome of Patients with Aortic Stenosis, Small Valve Area, and Low-Flow, Low-Gradient Despite Preserved Left Ventricular Ejection Fraction. Journal of the American College of Cardiology, 60, 1259-1267.

https://doi.org/10.1016/j.jacc.2011.12.054

[4] Lee, S.P., Kim, Y.J., Kim, J.H., Park, K., Kim, K.H., Kim, H.K., et al. (2011) Deterioration of Myocardial Function in Paradoxical Low-Flow Severe Aortic Stenosis: Two-Dimensional Strain Analysis. Journal of the American Society of Echocardiography, 24, 976-983. https://doi.org/10.1016/j.echo.2011.05.003

[5] Dayan, V., Vignolo, G., Magne, J., Clavel, M.A., Mohty, D. and Pibarot, P. (2015) Outcome and Impact of Aortic Valve Replacement in Patients with Preserved LVEF and Low-Gradient Aortic Stenosis. Journal of the American College of Cardiology, 66, 2594-2603. https://doi.org/10.1016/j.jacc.2015.09.076

[6] Hachicha, Z., Dumesnil, J.G., Bogaty, P. and Pibarot, P. (2007) Paradoxical Low-Flow, Low-Gradient Severe Aortic Stenosis Despite Preserved Ejection Fraction Is Associated with Higher Afterload and Reduced Survival. Circulation, 115, 2856-2864. 
https://doi.org/10.1161/CIRCULATIONAHA.106.668681

[7] Cavaca, R., Teixeira, R., Vieira, M.J. and Goncalves, L. (2017) Paradoxical Aortic Stenosis: A Systematic Review. Revista Portuguesa de Cardiologia, 36, 287-305.

https://doi.org/10.1016/j.repc.2016.09.010

[8] Abdul-Jawad Altisent, O., Puri, R., Regueiro, A., Chamandi, C., Rodriguez-Gabella, T., Del Trigo, M., et al. (2017) Predictors and Association with Clinical Outcomes of the Changes in Exercise Capacity after Transcatheter Aortic Valve Replacement. Circulation, 136, 632-643. https://doi.org/10.1161/CIRCULATIONAHA.116.026349

[9] Ozkan, A., Hachamovitch, R., Kapadia, S.R., Tuzcu, E.M. and Marwick, T.H. (2013) Impact of Aortic Valve Replacement on Outcome of Symptomatic Patients with Severe Aortic Stenosis with Low Gradient and Preserved Left Ventricular Ejection Fraction. Circulation, 128, 622-631.

https://doi.org/10.1161/CIRCULATIONAHA.112.001094

[10] Tribouilloy, C., Rusinaru, D., Marechaux, S., Castel, A.L., Debry, N., Maizel, J., et al. (2015) Low-Gradient, Low-Flow Severe Aortic Stenosis with Preserved Left Ventricular Ejection Fraction: Characteristics, Outcome, and Implications for Surgery. Journal of the American College of Cardiology, 65, 55-66.

https://doi.org/10.1016/j.jacc.2014.09.080 